\title{
Ergebnisse von Beobachtungen am Repsold'schen Registrirmikrometer \\ bei Anwendung eines Uhrwerks.
}

Von Dr. Fritz Cohn.

In Nr. 3719, Bd. I55 der A. N. hat Prof. H. Struve die Verbindung eines Uhrwerks mit dem sunpersönlichen Mikrometer von Repsold beschrieben, welches den Zweck hat, die Anwendung dieses Mikrometers bei Durchgangsbeobachtungen erheblich zu erleichtern, indem es den Haupttheil der Bewegung des Mikrometerfadens dem Beobachter abnimmt. Die von Prof. $H$. Struve in Gemeinschaft mit Dr. Rahts angestellten Versuche hatten die Vortheile des Uhrwerks gegenüber der gewöhnlichen Beobachtungsweise mit dem Repsold'schen Mikrometer, bei welcher die Bewegung des Mikrometerfadens ganz durch die Hand des Beobachters geleistet wird, uberzeugend dargethan und insbesondere gezeigt, dass seine Anwendung eine mindestens ebenso grosse, wenn nicht grössere Genauigkeit der Beobachtungen ermöglicht, sie für die verschiedenen Sterne gleichförmiger gestaltet und, was besonders wichtig ist, den Beobachter weit weniger anstrengt und ermudet, als es nach dem ubereinstimmenden Urtheile fast aller Beobachter sonst der Fall ist. Es erübrigte noch, die Vortheile dieser Beobachtungsweise durch ein grösseres Beobachtungsmaterial zu belegen, einige Untersuchungen von besonderem Interesse mehr im Einzelnen durchzuführen, sowie einige Bedenken, die sich bei den ersten Versuchen aufgedrängt hatten, näher zu prüfen. Als ich daher im Herbst 1900 an Stelle von Dr. Rahts die Beobachtungen am Repsold'schen Meridiankreise übernahm, war es mein Wunsch, die vorausgegangenen Untersuchungen in dieser Richtung zu vervollständigen. Die damals bevorstehende Eros-Opposition, an deren Beobachtung auch die hiesige Sternwarte sich betheiligte, liess die Verwirklichung dieser Absicht zunächst zurücktreten, da es gewagt schien, die neue Beobachtungsart ohne vorherige Uebung auf die Beobachtung der Eros-Vergleichsterne anzuwenden. Erst im Juli 1901, als eine längere Reihe klarer Abende derartige Untersuchungen begünstigte, konnten daher die Beobachtungen, auf welche sich die Prufung stützen sollte, in Angriff genommen werden. Das Folgende bildet einen Auszug aus einer ausführlicheren Darstellung, die später in den vVeröffentlichungen der Kgl. Universitätssternwarte zu Königsberg* erscheinen soll, und bezweckt, die ersten Ergebnisse dieser Prüfung vorzulegen und zu zeigen, in wie befriedigender Weise das Uhrwerk den Erwartungen entsprochen und die an die Einführung des. Repsold'schen Mikrometers geknüpften. Hoffnungen erfüllt hat.

Um zunächst auf das Bedenken, dem die Anwendung des Uhrwerks leicht ausgesetzt ist, näher einzugehen, so be- trifft dasselbe die Frage, ob dadurch nicht eine Aenderung des Collimationsfehlers eintreten könne, die es schwer sein würde, in Rechnung zu ziehen. Da nämlich das Uhrwerk mit seinen Gegengewichten doch über $3 \mathrm{~kg}$ wiegt, die nicht völlig symmetrisch vertheilt sind, so könnten derartige Einfluisse an sich wohl bestehen. Unsere bisherigen Untersuchungen haben indessen ergeben, dass der Collimationsfehler sowohl bei der Bestimmung aus Nadir, wie auch aus Mire ungeändert blieb, wenn man das Uhrwerk abnahm. Indessen ist zuzugeben, dass die bisherigen Versuche in dieser Richtung noch nicht abschliessend sind, da bei einer derartig gesteigerten Genauigkeit der Beobachtungen, wie sie sich im Laufe unserer Reihe herausstellte, auch viel grössere Anforderungen zu stellen sind, als dass man darüber nach so wenig Versuchen entscheiden könnte. Zudem bereitet die bedeutende Veränderlichkeit der Aufstellung des hiesigen Meridiankreises einer solchen Prüfung an sich schon grosse Schwierigkeiten. Mein Hauptbestreben richtete sich daher auf die Untersuchung der persönlichen Gleichung und ihr Verhalten bei längeren Beobachtungsreihen. Hierbei zog ich es vor, die Frage nach ihrer Abhängigkeit von der Declination, da sie sich am besten mit jener instrumentellen Prüfung verbinden würde, vorläufig auszuscheiden und nur die Fragen nach der Abhängigkeit der persönlichen Gleichung von der Zeit, der Disposition des Beobachters und der Helligkeit der beobachteten Sterne, diese aber möglichst eingehend, in Angriff zu nehmen, da hierzu allein die Beobachtung von Sternen innerhalb eines schmalen Declinationsgürtels erforderlich war. Auf diesen Punkt ist daher hier etwas näher einzugehen.

Zu den charakteristischen Fehlern der älteren Registrir-, ebenso wie der Auge und Ohr-Methode, zählt bekanntlich insbesondere die persönliche Gleichung des Beobachters. Während man anfangs wohl gehofft hatte, die persönliche Gleichung durch Registrirung der Durchgänge zum Verschwinden zu bringen, erwies es sich vielmehr alsbald, dass sie sogar grösseren Schwankungen im Laufe längerer Beobachtungsreihen, ja schon im Laufe eines Abends unterliegt und in jeder Beziehung von der Disposition des Beobachters und der Helligkeit des beobachteten Gestirns abhängt. Auf die schon im Laufe eines Abends, einer mehr. stündigen Beobachtungsreihe eintretende Aenderung der persönlichen Gleichung hat Küstner in seinen 》Beobachtungen von 4070 Sternen zwischen $0^{\circ}$ und $18^{\circ}$ nördlicher Declination*, Veröff. der Kgl. Sternwarte zu Bonn, Nr. 4, S. $30 \mathrm{ff}$. 
aufmerksam gemacht uud seine Erfahrungen in Nr. 5 derselben Veröffentlichungen, S. 4, bestätigt gefunden. Danach ändert sich seine persönliche Gleichung stündlich um 0.520 , welchen Betrag er den > Gang des Beobachters Ebenso erfährt sie bei jeder Unterbrechung der Beobachtungen eine Aenderung. Ferner ist bekannt, dass die Hellig. keitsgleichung vom-Luft-und-Bildzustande abhängig ist (siehe z. B. Battermann, »Resultate aus Beobachtungen von 379 Anhaltsternen und 1640 durch Anschluss bestimmten Sternen $\%$, Beob.-Ergebnisse der Kgl. Sternwarte zu Berlin, Heft Nr. 8, S. 15 u. I6). Die Einführung des Repsold'schen sunpersönlichen. Mikrometers sollte vor Allem den erwähnten Uebelständen abhelfen, indem bei der Bisection eines Sternbildes, als welche sich die Beobachtung bei einigermaassen guten Luftverhältnissen thatsächlich darstellt, doch nur Auffassungsunterschiede von wenigen Zehntel Bogensecunden auftreten können. In der That wird nach den bisherigen Erfahrungen die persönliche Gleichung stark heruntergedrückt, siehe z. B. Albrecht, $\gg$ Die Beobachtungsmethode mittelst des Repsold' schen Registrirmikrometers in ihrer Anwendung auf Längenbestimmungene, A. N. Bd. 155, Nr. 3699. Ueber den Effect der Helligkeit bei der bisherigen Anwendung des Repsold' schen Mikrometers sind mir keine Zahlenangaben bekannt, nur berichtet Prof. Seeliger in der V.J.S. für 1900, S. 142, dass bei den Beobachtungen in München sich mit vollkommener Sicherheit die Thatsache herausgestellt habe, dass beim Repsold'schen Registrirmikrometer eine mit der Helligkeit der beobachteten Sterne wechselnde persönliche Auffassung des Beobachters nicht nachzuweisen iste. Uebrigens würde man, selbst wenn dieses der Fall sein sollte - und dass auch bei Bisectionen von Sternscheibchen systematische, von der Grösse des Scheibchens abhängige Fehler bestehen können, ist aus der Ausmessung photographischer Aufnahmen bekannt - versuchen können, diesen Fehler durch Anwendung eines Reversionsprismas unschädlich zu machen; doch werden wir alsbald sehen, dass er bei unseren Beobachtungen auch ohnedies unmerklich war. Wenn nun schon bei der gewöhnlichen Beobachtung mit dem Repsold'schen Mikrometer derartige Erfolge erzielt waren, so war bei Anwendung des Uhrwerks, welches der Beobachtung einen ganz anderen Grad von Bequemlichkeit verleiht, eine noch weitergehende Constanz der persönlichen Gleichung sicher zu erwarten.

Da es sich nicht um die Untersuchung der absoluten persönlichen Gleichung, sondern nur ihrer Schwankungen handelte, unternahm ich zusammen mit dem Rechner der Sternwarte, Herrn stud. math. A. Postelmann, der sich auch im Weiteren an den höchst zeitraubenden Ablesungen der Chronographenstreifen in dankenswerther Weise betheiligte, die gemeinsame Beobachtung einer grösseren Liste von Sternen. Dieselbe erstreckte sich, um alle auftretenden Fragen mit der wünschenswerthen Entschiedenheit beantworten zu können, über nahezu 4 Rectascensionsstunden, von $16^{\mathrm{h}} 17^{\mathrm{m}}$ bis $19^{\mathrm{h}} 5^{6 \mathrm{~m}}$, und enthielt fast 100 Sterne, die an einer grösseren Reihe von Abenden durchbeobachtet werden sollten. Die grosse Mehrzahl der Sterne lag zwischen $+15^{\circ}$ und $+20^{\circ}$ Declination; nur wenn Lücken auftraten - wir konnten nicht unter $8{ }^{m}$. hinuntergehen und wollten in kurzer Zeit ein möglichst grosses Beobachtungsmaterial sammeln - wurden etwas südlichere Sterne bis $z u+10^{\circ}$ hinzugezogen. Auch gehen einige Zeitsterne, deren Mitnahme der Ableitung definitiver Sternpositionen wegen erwtinscht, wenn auch der Frage selbst wegen nicht nothwendig war, über diese Grenzen noch hinaus. Der Helligkeit nach vertheilten sich die Sterne möglichst über alle der Beobachtung zugänglichen Grössenclassen, von den heHsten-Sternen wie $\alpha$ Aquilae und $\alpha$ Ophiuchi bis $z u$ Sternen $\mathbf{8}^{\mathrm{m}} \cdot 5$. Unter diese letztere Helligkeitsgrenze hinabzugehen, erwies sich nach einigen Versuchen als unthunlich, indem bei der Bisection wenigstens eine Andeutung eines Lichteffects übrig bleiben muss. Es kann dies als ein geringfügiger Mangel der Beobachtungen mit dem Repsold'schen Mikrometer angesehen werden, der z. B. Küstner vorzugsweise veranlasst hat, seine erwähnten Beobachtungen nach der gewöhnlichen Registrirmethode anzustellen. Während ich im vorigen Winter Sterne $9 . \mathrm{m}_{2}$ bis $9 \cdot 3$ bei vierzölligem Objective und fast 200 facher Vergrösserung noch leidlich sicher registriren konnte, konnten wir im Juli dieses Jahres Sterne $\mathbf{8}^{\mathbf{m}} \mathbf{5}$ nur mit grösster Anstrengung beobachten und meinten schon von $\mathbf{8}_{.2}^{\mathrm{m}}$ ab eine Abnahme der Sicherheit zu verspüren, was librigens die Ergebnisse nicht bestätigt haben. Inzwischen ist es mir aber gelungen, durch Anwendung einer schwächeren, etwa 120 fachen Vergrösserung, welche die Genauigkeit kaum vermindert haben dürfte, die untere Grenze um $0^{\mathrm{m}} 7$ bis $0^{\mathrm{m}} .8$ herunterzudrücken und im September und October Sterne $9 \mathrm{~m}_{2}$ bis $9 \cdot 3$ noch ziemlich sicher zu beobachten und jedenfalls weit sicherer, als bei den vorjährigen Registrirbeobachtungen. Wieviel an diesem Erfolg der durchsichtigeren Luft und der grösseren Dunkelbeit der Herbstabende, wieviel der schwächeren Vergrösserung zuzuschreiben ist, kann dahingestellt bleiben; jedenfalls gab ich darauf den Versuch, die schwächsten Sterne mit einem Doppelfaden zu beobachten, bald auf, denn ich glaube nicht, dass man von einem vietzölligen, mehr als 60 Jahre alten Objective und bei Feldbeleuchtung mehr wird verlangen können. Gerade bei diesen schwächsten Sternen bietet übrigens die Anwendung des Uhrwerks erhebliche Vortheile, indem stets die angespannteste Aufmerksamkeit auf diejenige Stelle gerichtet bleiben kann, an der sich das Lichtpünktchen befindet, und nicht durch das Drehen der Mikrometerschraube abgelenkt wird. Jedenfalls glaube ich nicht, dass man derartig schwache Sterne ohne Uhrwerk beobachten kann.

Das Schema der Beobachtungen war derartig angelegt, dass gleichzeitig die beiden Methoden zur Bestimmung der relativen persönlichen Gleichung zur Anwendung gelangten, sowohl diejenige, bei welcher die Beobachter während eines Sterndurchganges abwechseln (Gruppe I von 38 Sternen), als auch diejenige, bei der jeder Stern von einem Beobachter durchbeobachtet wird, die Beobachter aber von Tag zu Tag mit den Sternen abwechseln (1 2 Gruppen von je 5 Sternen, von denen jeder Beobachter abwechselnd eine Gruppe beabachtete). Die Sterne der ersten Gruppe mussten, um den Beobachtern Zeit zum Wechseln zu lassen, während zweier etwa je $20^{5}$ langer und durch eine ebenso lange Pause getrennter Intervalle beobachtet werden. Auch hierbei machte sich die Anwendung des Uhrwerks, das inzwischen sehr nahe der Bewegung des Sterns gefolgt war, vortheilhaft geltend. Aber auch die Beobachtung der zweiten Gruppe von Sternen 
wurde in derselben Art auf zwei Iatervalle mit einer Pause ausgedehnt, die einmal zum Ausruhen diente, etwaige Aenderungen der Beleuchtung oder der Geschwindigkeit, die während der Messung ausgeschlossen sind, ermöglichte, und endlich, was das Wesentlichste war, die Helligkeitsgleichung durch Anwendung einer Kappe während eines der beiden Intervalle zu bestimmen gestattete. Bei beiden Gruppen wurde, sobald die Helligkeit $6^{\text {th }}$ uberstieg, eine etwa $2^{1 / 2}$ Grössenclassen abschwächende Kappe aus feinmaschigem Drahtnetz möglichst abwechselnd während des ersten oder zweiten Intervalls und für die beiden Beobachter angewandt.

Was die Beobachtungsweise mit dem Uhrwerk an sich anlangt, so ist der Anzeige von Prof. $H$. Struve wenig mehr hinzuzufügen. Der Mikrometerfaden wird ein Stiick (ca. 20s) vor die durch einen festen Faden oder sonstwie markirte Stelle gebracht, von der an die Beobachtung beginnen soll, die Geschwindigkeitsscala der Declination entsprechend eingestellt und das Uhrwerk, sobald der Stern an den Faden tritt, in Bewegung gesetzt. Wenige Secunden genligen, um zu zeigen, ob die Einstellung der Scala genau genug war, oder sie aus freier Hand an einer Handhabe zu verbessern. Dann beginat die exacte Beobachtung, indem man durch ständiges Pointiren auf den Stern die kleinen Unregeimässigkeiten des Uhrwerks etc. zu beseitigen sucht. Wie jede neue Beobachtungsmethode, so erfordert auch diese natürlich eine gewisse Uebung. Trotzdem erlangt man darin schon nach wenigen Sternen eine bedeutende Fertigkeit, sodass verschiedene gelegentliche Besucher der Sternwarte, u. a. Herr Prof. Ludwig Struve, sehr bald die Methode anwenden konnten. Auch Herr Postelmann hatte beim Beginn unserer gemeinsamen Beobachtungen erst wenig Uebungsversuche angestellt. Nach einiger Uebung gelingt es fast stets, alle Sterne an derselben Stelle der Mikrometerschraube zu beobachten, ja, sie längere Zeit, 1/2 Minute und mehr, obgleich das kaum jemals erforderlich sein dürfte, genau auf dem Faden zu halten. Eine völlige Beherrschung des Uhrwerks und seiner Wirkungsweise erlangt man freilich erst nach längerer Zeit, und es ist sicher, dass wir erst im Verlaufe der vorliegenden Beobachtungen dahin gelangt sind, ja vielleicht überhaupt noch vicht die ganze Leistungsfähigkeit der Methode erschöpft haben. Erwähnt sei noch, dass die vierstiundige ununterbrochene Beobachtung von roo Sternen uns niemals irgendwie merklich ermüdet hat, sodass wir am Schluss eine Abnahme der Genauigkeit hätten befürchten müssen.

In dieset Weise wurde die etwähnte Sternliste an einer grösseren Zahl von Abenden des Juli durchbeobachtet, indessen war es besonders in der ersten Zeit nur während eines Theils der Liste klar, und auch sonst wurden die Beobachtungen vielfach durch Wolken gestört, sodass nur an etwa zehn Abenden eine fortlaufende Reihe erhalten würde. An den letzten vier dieser Abende musste aber der Anfang der Liste, weil in den Tag gelaufen, fortgelassen werden, sodass die Gesammtzahl der an 15 Abenden erhaltenen Beobachtungen sich nur auf rund 1000 stellt und sonach im Durchschnitt für jeden Stern etwa ro beträgt. Zu erwähnen ist noch, dass an den beiden letzten Tagen die Sterne beider Gruppen auf die erste Art, unter ständigem Wechsel der Beobachter während jedes Durchgangs, beobachtet wurden, um für einea $\Lambda$ bend eine grössere Zahl von directen Bestimmungen des Auffassungsunterschiedes zu erhalten. Der Luftzustand war nach dem Obigen anfangs kein sehr be. friedigender, nur an den späteren Abenden ist er als gut bezeichnet, sodass nach unserem Erachten der durchschnitt. liche Luftzustand während unserer Beobachtungen kaum als normal bezeichnet werden kann. Dementsprechend glückten auch an den ersten Abenden die Beobachtungen nach unserer Empfindung nur mässig und genügten jedenfalls nicht völlig den Erwartungen, die wit an die Methode geknupft hatten. Denn da jedes gelegentliche, der Unvollkommenheit des Uhrwerks oder der Unruhe der Luft entspringende Abweichen des Sternbildes vom Faden die Ruhe des Beobachters, von der das Gelingen der Beobachtung wesentlich abhäugt, empfindlich stört, so haben ungünstige Luftverhältnisse, indem sie die Disposition des Beobachters verschlechtern, einen doppelt ungünstigen Einfluss auf die Genauigkeit der Messungen. Erst allmählich gewöhnt man sich die nöthige Ruhe an, und eben in der Aneignung dieser Ruhe beruht die durch Uebung gesteigerte Leistungsfähigkeit der Methode.

Das Ablesen der Streifen, das wohl der ermüdendste Theil der ganzen Arbeit war, - es waren im Ganzen fast 30000 Signale abzulesen - erstreckte sich nicht auf alle in die betreffenden Intervalle fallenden Contacte, sondern nur auf jeden zweiten, was bei der schnellen Aufeinanderfolge (je $0^{9} 6$ ein Contact) eine völlig geaügende $Z$ ahl von Beob. achtungsmomenten lieferte. In jedes Intervall fielen bei der ersten Gruppe von Sternen I 5 , bei der zweiten I 3 Signale, sodass eine vollständige Sternbeobachtung auf 30 resp. 26 Contacten beruht, die bei dem ununterbrochenen Pointiren als ziemlich unabhängig vou einandet gelten können.

Die Berechnung musste hier etwas umständlicher werden, als es an sich erforderlich ist, indem, um einmal den persönlichen Auffassungsunterschied, dann im Falle der Kappenbeobachtungen die Helligkeitsgleichung zu erhalten, die beiden Theile der Beobachtung für sich auf die Mitte reducirt werden mussten.

Wir gehen nun zur Besprechung der Resultate über. Für den persönlichen Auffassungsunterschied konnte man aus dem Beobachtungsmaterial zwei ganz unabhängige Bestimmungen ableiten. Einmal ergab die erste Gruppe von Sternen unmittelbar den Werth $C-P$ (neben einer Verbesserung des angenommenen Schraubenwerthes), woraus dann Abendmittel gebildet wurden. Dann aber konnte man durch Vergleichung zweier aufeinanderfolgender Abende aus der zweiten Gruppe von Sternen die relative persönliche Gleichung im Mittel beider Abende erhalten. Um indessen das ganze Material verwerthen zu können, wurde diese zweite Bestimmung in etwas modificirter Form ausgefuhrt. Es wurden nämlich in der üblichen Art unter Zugrundelegung der 2 r mitbeobachteten Jahrbuchsterne - mit Hinzufügung der Auwers' schen *Vorläufigen Verbesserungen stern mitbeobachtet und die Aufstellung des Fernrohrs wiederholt bestimmt worden - die definitiven Sternörter für den Jahresanfang abgeleitet, was ja auch sonst aus verschiedenen Gründen nahe lag. Das Mittel aller Bestimmungen eines Sterns wird infolge des stetigen Abwechselns der Beobachter von der Differenz $C-P$ unabhängig und auf das Mittel $1 / 2(C+P)$ bezogen sein; fällt die Differenz nicht völlig 
aus dem Mittel heraus, so genugt es jedenfalls, einen leicht provisorisch zu ermittelnden Werth dafur anzuwenden. Fasst man nun wieder die Abweichungen der Einzelpositionen von diesem Endwerthe für den Abend und für jeden Beobachter in Mittelwerthe zusammen, so erhält man wieder die abendliche Differenz $C-P$ für die Sterne der zweiten Gruppe, so gut wie unabhängig von den erhaltenen Endpositionen der Sterne und vom Uhrgange. Der Sicherheit halber wurde der letztere noch für jeden einzelnen Abend mit Berücksichtigung sämmtlicher Sterne gepruft, was aber nur in zwei Fallen zu Aenderungen Anlass gab; im Uebrigen konnte es bei der linearen Ausgleichung der Jahrbuchsterne sein Bewenden haben. Die Uebereinstimmung der beiden so futr jeden Abend erhaltenen, von einander gánzlich unabhängigen Bestimmungen der Differenz $C-P$ wird alsbald einen wesent- lichen Beweis für die Constanz dieses Werthes im Laufe eines jeden Abends abgeben.

In der folgenden Zusammenstellung, welche die Ergebnisse dieser ersten Prüfung des persönlichen Auffassungsunterschiedes enthält, sind die für $C-P$ erhaltenen Abendwerthe für beide Sterngruppen neben einander gegeben, ebenso ihr einfaches Mittel; alle Werthe mit Angabe der Zahl der Sterne, auf det sie beruhen. Die schliesslichen Mittelwerthe aus der ganzen Reihe sind ohne Berücksichti. gung des verschiedenen Gewichts der einzelnen Abendwerthe abgeleitet. Für die Tage, an denen die Sternzahl der einzelnen Gruppen zu gering erschien, um einen sicheren Werth fur $C-P$ zu liefern, ist nur das Mittel aller Sterne gegeben.

\begin{tabular}{rr||c|c|c|c|c|c}
\multicolumn{1}{r||}{} & \multicolumn{2}{c|}{ Gruppe I. } & \multicolumn{2}{c|}{ Gruppe II. } & \multicolumn{2}{c}{ Mittel } \\
\hline \multirow{2}{*}{ I901 } & $\begin{array}{c}\text { Directe Bestimmung } \\
C-P\end{array}$ & Sterne & $\begin{array}{c}\text { Indirecte } \\
C-P\end{array}$ & Sestimmung & $C-P$ & Sterne \\
\hline \multirow{2}{*}{ Juli } & I & -0.020 & 17 & - & - & -0.021 & 30 \\
& 2 & -0.004 & 34 & -0.016 & 55 & -0.010 & 89 \\
& 3 & -0.026 & 36 & -0.030 & 48 & -0.028 & 84 \\
& 5 & -0.014 & 30 & -0.016 & 19 & -0.015 & 49 \\
6 & -0.020 & 35 & -0.029 & 58 & -0.025 & 93 \\
9 & -0.026 & 24 & -0.023 & 20 & -0.024 & 44 \\
I0 & -0.032 & 34 & -0.021 & 60 & -0.026 & 94 \\
I1 & - & - & - & - & $-0.033:$ & 28 \\
I2 & -0.019 & 36 & -0.020 & 59 & -0.020 & 95 \\
I3 & -0.019 & 35 & -0.024 & 54 & -0.022 & 89 \\
I5 & -0.020 & 30 & -0.020 & 39 & -0.020 & 69 \\
I9 & -0.033 & 27 & -0.030 & 40 & -0.031 & 67 \\
22 & -0.013 & 65 & - & - & -0.013 & 65 \\
23 & -0.016 & 65 & - & - & -0.016 & 65 \\
\hline Mittel & -0.020 & \pm 0.0024 & -0.023 & \pm 0.0016 & -0.022 & \pm 0.0018
\end{tabular}

Eine derartige Constanz des Auffassungsunterschiedes dürfte bisher kaum jemals auch nur annähernd erreicht sein und lässt die Leistungsfähigkeit der neuen Methode im hellsten Licht erscheinen. Aus der Vergleichung mit dem Endwerth von -0.022 folgt als mittlerer Fehler eines Abendwerthes \pm 0.007 , während auf Grund der Einzelwerthe, die $z u$ den Abendmitteln zusammengewirkt haben, etwa $\pm 0.005 \mathrm{zu}$ erwarten gewesen wäre. Dass sich ein geringfügiger Unterschied beider Beobachter im Betrage von o"3 als reell herausgestellt hat, darf nicht weiter Wunder nehmen. Einmal kann die absichtlich ein wenig zu langsam eingestellte Bewegung der Mikrometerschraube und das dadurch bedingte beständige leise Nachdrehen seitens des Beobachters einen derartigen individuellen Unterschied zur Folge haben. Würde man die Scala abwechselnd auch auf eine etwas zu grosse Geschwindigkeit einstellen, so muisste man mit der Hand durch Reibung der Bewegung des Uhrwerks entgegenwirken, was uns wenigstens nicht in der erforderlichen Feinheit gelang. Ferner könnte mitwirken, dass, da der eine Beobachter ziemlich kurzsichtig, der andere normalsichtig ist, das Ocular beim Wechsèl der Beobachter stets verstellt werden musste, was auf die Auffassung des nicht genau in der Mitte des Gesichtsfeldes befindlichen Mikrometerfadens einen kleinen
Einfluss gehabt haben kann. Die kleinen Schwankungen, die man vielleicht noch in den obigen Werthen bemerken kann, whirden sich danach durch veränderten Luftzustand, ungleichartigen Gang des Uhrwerks und verschiedene Disposition des Beobachters leicht erklk̈ren lassen.

Ferner zeigt die persobnliche Differenz trotz der erst allmählich erlangten Uebung der beiden Beobachter keine Spur einer zeitlichen Aenderung. Durch Zusammenfassung mehrerer Abende in ein Mittel erhkilt man die folgenden Werthe :

$$
\begin{aligned}
& \text { I901 direct } C-P \text { indirect } \\
& \text { Juli 2-6 -0.016 -0.023 } \\
& \text { 9-13 }-0.024 \quad-0.022 \\
& \begin{array}{lll}
15-23 & -0.020^{\circ} & -0.025
\end{array}
\end{aligned}
$$

Ebensowenig zeigt sich eine Schwankung im Laufe des Abends, wie sie durch eine mehr oder minder grosse Abspannung der Beobachter sich erklären liesse. Zieht man in der später gegebenen Uebersicht der von den Beobachtern erhaltenen Endwerthe für jeden Stern je zehn Sterne in ein Mittel zusammen, so erhalt man folgende Zahlen : 


$\begin{array}{ll}-0.024 & -0.019 \\ -0.026 & -0.023 \\ -0.024 & -0.024 \\ -0.021 & -0.009 \\ -0.015 & -0.022\end{array}$

In dem Gesammtmaterial wenigstens ist sonach keine Spur einer Aenderung im Laufe der zunehmenden Rectascensionen bemerkbar. Eine diesbezügliche Discussion der einzelnen Abende vornehmen zu wollen, würde wohl doch die Beweiskraft der Beobachtungen übersteigen.

Auf Grund dieser Ergebnisse wird man daher behaupten dürfen, dass die persönliche Gleichung der beiden Beobachter im Laufe einer mehrwöchentlichen Beobachtungsreihe innerhalb der Grenzen von os.o1 unverändert geblieben ist.

Wir gehen nun über zu den auf die Helligkeitsgleichung bezüglichen Untersuchungen und können auch diese wieder in mehrfacher, von einander unabhängiger Weise ableiten. Eine directe Bestimmung des Effects der Kappe geben hier die Sterne der zweiten Gruppe, soweit sie heller als $6^{\mathrm{m}}$ sind. $\mathrm{Da}$ das aber nur bei 28 der 60 Sterne zutrifft und von diesen wieder nur die Hälfte auf jeden einzelnen Beobachter fullt, so ist das Material nur an wenigen Tagen umfangreich genug, um einen einigermaassen sicheren Werth des Kappeneffects zu ergeben. Trotz der relativen Unsicherheit der im Folgenden zusammengestellten Abendwerthe wird man aber doch sagen können, dass kaum Andeutungen eines solchen Einflusses an einzelnen Abenden vorhanden sind.

\begin{tabular}{rr|c|c|c|c}
\hline \multicolumn{1}{r|}{ 1901 } & \multicolumn{1}{c|}{$K_{C}$} & Zahl & $K_{P}$ & Zahl \\
\hline Juli & 2 & +0.001 & 8 & +0.007 & 14 \\
& 3 & -0.004 & 11 & 0.000 & 8 \\
6 & +0.002 & 15 & +0.001 & 12 \\
10 & -0.014 & 14 & -0.001 & 11 \\
12 & -0.011 & 14 & -0.011 & 13 \\
13 & -0.013 & 8 & +0.007 & 12 \\
15 & +0.008 & 10 & -0.015 & 8 \\
19 & -0.001 & 10 & +0.001 & 7 \\
\hline Mittel & -0.004 & & -0.001 &
\end{tabular}

Durch Zusammenfassung aller Einzelbestimmungen, auch der kürzeren Abendreihen, in ein Mittel, erhält man als Kappeneffect

$$
\begin{array}{ccc}
\text { aus } 105 \text { Sternen für } C: & -0.0028 \pm 0.0029 \\
, 104 * \# \quad P: & 0.0000 \pm 0.0030
\end{array}
$$

also Werthe, die angesichts ihrer mittleren Fehler als verschwindend anzusehen sind.

Eine indirecte Bestimmung erhält man aus den Sternen der ersten Gruppe, wenn man für jeden Stern je zwei Endwerthe, $C$ und $P$, mit und ohne Kappe, bildet und mit einander vergieicht. ${ }^{3}$ ) Auf diesem Wege findet man als Kappeneffect :

$$
\begin{gathered}
\text { aus } 13 \text { Sternen für } C:-0.0021 \pm 0.0034 \\
, 12, \quad P:+0.0036 \pm 0.0107,
\end{gathered}
$$

wieder fast völlig verschwindende Werthe. Auch eine Anordnung dieser Werthe nach der Helligkeit der Sterne gab keine Veranlassung, die vollständige Einflusslosigkeit der Kappe zu bezweifeln.

Endlich erhalten die angefuhrten Ergebnisse der Prlifung der Helligkeitsgleichung eine Ergänzung und Bestätigung durch die directe Vergleichung der von den beiden Beob. achtern für sich erhaltenen Endpositionen der Sterne und ihre Anordnung nach der Helligkeit. Dabei wurden die Helligkeiten der Sterne uber $7 \mathrm{~m}_{5}$ der Potsdamer Durchmusterung entnommen, während die schwächeren Sterne von beiden Beobachtern wiederholt und in recht guter Uebereinstimmung geschătzt worden waren. Auf diesem Wege war auch, nachdem man sich von der Gleichartigkeit beider Scalen überzengt hatte, die Abschwächung der Kappe zu 2.5 Grössenclassen bestimmt worden. Die Anordnung der

\begin{tabular}{|c|c|c|c|c|}
\hline Helligkeit & \multicolumn{2}{|c|}{$C-P$} & \multicolumn{2}{|c|}{$\begin{array}{c}\text { Zahl } \\
\text { der Sterne }\end{array}$} \\
\hline $\begin{array}{c}>4^{\mathrm{m}} \cdot 0 \\
4^{\mathrm{m}} \mathrm{I}-5^{\mathrm{m}} \cdot \mathrm{o}\end{array}$ & $\begin{array}{l}-0.014 \\
-0.020\end{array}$ & -0.018 & $\begin{array}{r}7 \\
\times 6\end{array}$ & 23 \\
\hline $\begin{array}{l}5.1-6.0 \\
6.1-7.0\end{array}$ & $\begin{array}{l}-0.024 \\
-0.016\end{array}$ & -0.020 & $\begin{array}{l}18 \\
14\end{array}$ & $3^{2}$ \\
\hline $\begin{array}{l}7.1-7.5 \\
7.6-7.9 \\
8.0-8.1\end{array}$ & $\begin{array}{l}-0.023 \\
-0.023 \\
-0.023\end{array}$ & -0.023 & $\begin{array}{l}17 \\
29 \\
15\end{array}$ & 46 \\
\hline $\begin{array}{l}8.2-8.3 \\
8.4-8.5\end{array}$ & $\begin{array}{l}-0.023 \\
-0.014\end{array}$ & -0.021 & $\begin{array}{r}17 \\
7\end{array}$ & 39 \\
\hline
\end{tabular}
Werthe $C-P$ nach der Helligkeit - im Falle der Kappe gab es für jeden Stern zwei solche Werthe - hatte das folgende Resultat:

Sieht man von den beiden äussersten Werthen, die auf einer ganz geringen Zahl von Sternen beruhen, ab, so kann man inverhalb der Grenzen von $0^{5} .001$ bis 0.002 von einer völligen Constanz der Auffassung sprechen; eine relative Helligkeitsgleichung ist also nicht im geringsten nachzuweisen.

Wenn ich nun auch das vorliegende, im Laufe dreier Wochen und hauptsächlich zu anderem Zweck gesammelte Material für die Untersuchung der Helligkeitsgleichung im Einzelnen, sobald man die allergrösste Genauigkeit in den o.00 I erstrebt und auch die Sterne der ersten vier Grössenclassen strenger einbeziehen will, noch nicht als völlig ausreichend erachte, so ist doch soviel sicher, dass sie den Betrag von 0.00 I "für eine Grössenclasse keinesfalls überschreiten kann.

Dadurch erlangt man gegenüber den bisher bei Registrirbeobachtungen angewandten Bestimmungen der Helligkeitsgleichung einen grossen Vortheil.

Erst seit kurzer Zeit, seit kaum zwei Jahrzehnten, hat man angefangen, der Helligkeitsgleichung und der Elimination ihres Einflusses auf registrirte Sterndurchgänge grössere Auf-

1) Da die Reihenfolge der Beobachter bei diesen Sternen von Tag zu Tag wechselt, so wechselt auch für jeden Beobachter der Gebrauch der Kappe, z. B.:

$$
\begin{array}{cll} 
& \text { Intervall I } & \text { Intervall II } \\
\text { Juli } 2 & C \text { ohne Kappe } & P \text { mit Kappe } \\
3 & P & C
\end{array}
$$


merksamkeit zu schenken, indem man die helleren Sterne durch ein vor das Objectiv gebrachtes Gitter abschwächte und den Effect dieser Abschwächung gegenuber der $\mathrm{Be}$ obachtung des ungeschwächten Sternbildes bestimmte. Dabei begnügte man sich anfangs damit, diesen an einzelnen Abenden bestimmten Betrag für die ganze Reihe anzuwenden; indessen ergaben diese verschiedenen Versuche nicht immer die gentigende Uebereinstimmung, sodass man vorlaufig fast stets von einer Berlicksichtigung der Helligkeitsgleichung Abstand genommen hat. Die Ursache ist unzweifelhaft die, dass die Helligkeitsgleichung ganz wesentlich vom Luft- und Bildzustande abhängt; ihre gelegentliche Bestimmung ist daher nur ein Nothbehelf, und so stellt z. B. Battermann in seiner früher citirten Arbeit (S. I6) als unumgängliche Forderung für klinftige Beobachtungsreihen auf, dass die Helligkeitsgleichung bei der Beobachtung selbst eliminirt werde, indem systematisch alle Sterne möglichst auf eine gleiche Helligkeit abgeschwächt witrden. Indessen bietet auch dieses Verfahren, welches wohl zum ersten Male von Küstner bei seiner erwähnten .Beobachtungsreihe am Bonber Meridiankreise wirklich durchgefuhrt worden ist, manche Bedenken oder wenigstens Schwierigkeiten. Einmal ist die Erzielung gleicher Helligkeit mit einer geringen Zahl von Kappen doch nur in beschränktem Maasse möglich - bei Küstner schwankt die abgeschwächte Helligkeit etwa zwischen $7^{\text {mo }}$. und $10 \mathrm{mo}$, und ist im Mittel $8^{\mathrm{m}} \cdot 5$-, sodass immer noch eine Discussion fur diesen Bereich, in welchem gerade die Helligkeitsgleichung oftmals sich besonders geltend macht, vorzunehmen ist. Andernfalls wäre es auch äusserst unbequem, wenn alle Sterne den schwächsten, die man noch beobachten will, angenähert würden. Der Mühe einer genauen Grössenschätzung, insbesondere bei verschleiertem Himmel, und der Discussion der Helligkeitsgleichung wird man doch nicht völlig ubberhoben, zumal wenn sie grössere Beträge als bei Küstner erreicht oder gar unregelmässig verläuft. Alle diese Schwierigkeiten fallen für die Beobachtungen mit dem Repsold'schen Mikrometer nach unseren Ergebnissen fort. Wird man es auch im Interesse einer schärferen Pointirung gewiss vorziehen, die allzu hellen Sterne abzuschwächen, so ist doch weder eine scharfe Grössenschätzung, noch mehr als eine gelegentliche Bestimmung des Effects der $\mathrm{Ab}$. schwächung erforderlich. Auch der Luftzustand übt, abgesehen von einer Erhöhung der Unsicherheit, keinen systematischen Einfluss mehr aus.

Sind somit die Ergebnisse, soweit sie die Unveränderlichkeit der persönlichen Gleichung wăhrend längerer Zeiten und ihre Unabhängigkeit von der Helligkeit betreffen, schon sehr befriedigęnd, so ist auch die individuelle Genauigkeit der Beobachtungen eine bedeutend grössere geworden, als sie sonst bei Meridianbeobachtungen erreicht worden ist. Schon bei der Ableitung der Werthe $C-P$ erhielt man hierfür einen deutlichen-Beweis. Hier mögen $z$ wei andere Bestimnungen des mittleren Fehlers einer Beobachtung angefuhrt werden. Die eine leitet ihn für jeden Abend und für jeden Beobachter aus den Abweichungen der Positionen des betreffenden Abends von den definitiven Sternpositionen ab; die Unsicherheit dieser letzteren, die durchschnittlich auf etwa ro vollständigen Beobachtungen beruhen, wurde dadurch näherungsweise berücksichtigt, dass die erhaltenen Werthe um $1 / 18$ vermehrt wurden. Der Zweck dieser Bestimmung ist die.Vergleichung der verschiedenen Abende und der beiden Beobachter. Bei der anderen Bestimmung vergleicht man in der liblichen Art die Einzelpositionen eines jeden Sterns mit dem Endwerthe und erhält so für jeden einzelnen Stern den mittleren Fehler einer Beobachtung, wodurch eine. Anordnung nach. der Helligkeit der Sterne ermöglicht wird. Wir fuhren hier zunächst die Ergebnisse der ersten Rechnung an. Für die Sterne der ersten Gruppe ist der mittlere Fehler des von dem einzelnen Beobachter beobachteten halben Durchgangs $\left(\varepsilon_{C}\right.$ und $\left.\varepsilon_{P}\right)$ gegeben; daneben $\varepsilon_{1 / 2}(C+P)$, der mittlere Fehler der vollständigen Beobachtung. Bei den Sternen der zweiten Gruppe waren nur die mittleren Fehler für jeden Beobachter anzugeben. In der letzten Columne sind dann alle Sterne in Eins zusammengezogen.

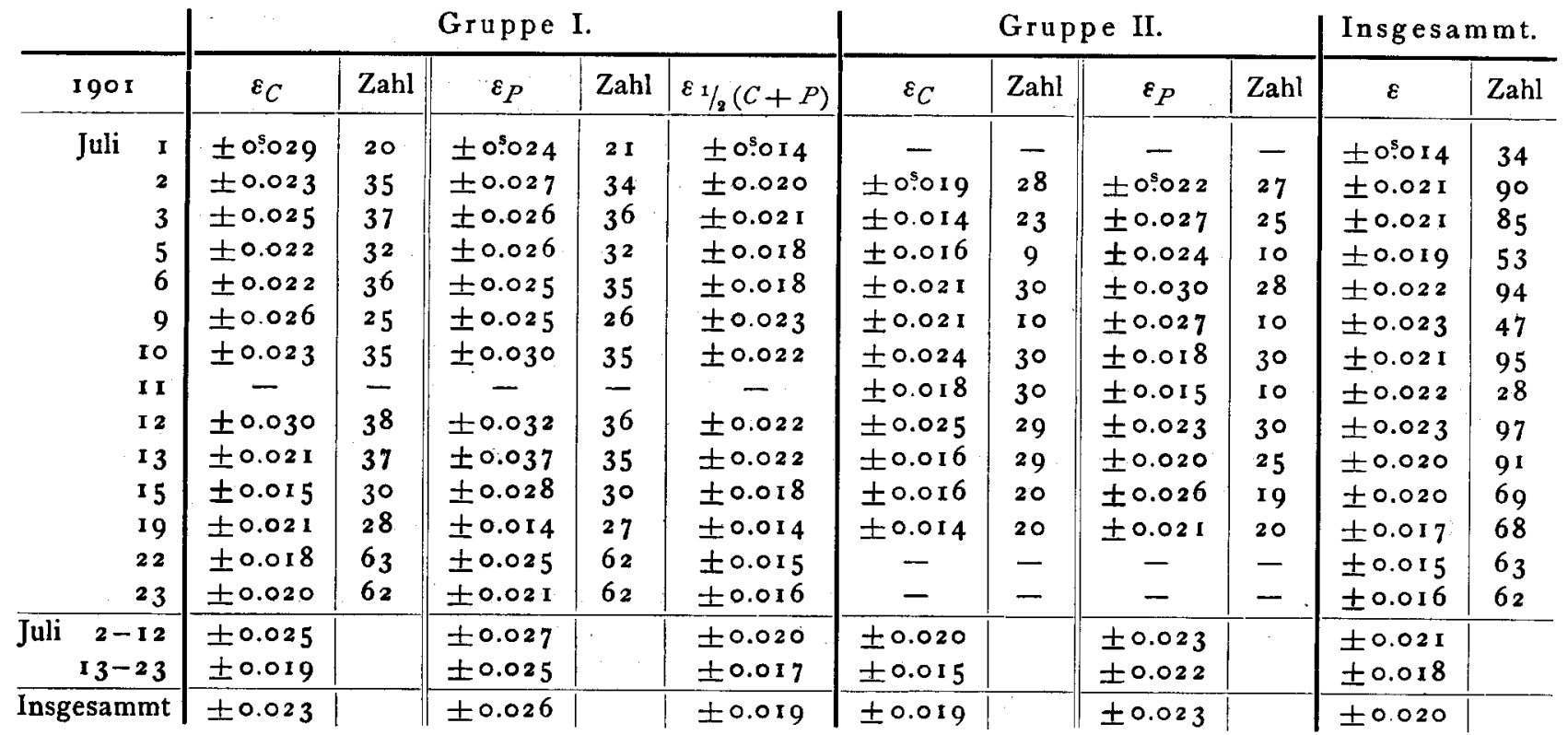


Aus der anfänglichen bemerkenswerthen Constanz der Zahlen der letzten Columne ersehen wir zunächst - von dem mehr zufälligen Werth für Juli I können wir absehen -, wie selbst Beobachter, die in dieser Beobachtungsart noch ungeübt sind, sehr schnell eine bedeutende Genauigkeit erlangen. Erst an den drei letzten Abenden tritt eine erhebliche Steigerung der Genauigkeit ein. Zum Theil wird dies darauf zurückzuführen sein, dass das Uhrwerk, dessen Gang unregelmässig geworden war, vor dem r 9 . Juli gereinigt wurde und nun viel leichter der corrigirenden Hand des Beobachters nachgab. Indessen scheint diese Erklärung mehr fur den Beobachter $P$ als für $C$ zuzutreffen, indem die Werthe $\varepsilon_{C}$ auch schon am 13. und 15. Juli, an denen gerade das Haken des Uhrwerks bemerkbar wurde, bedeutend ab. genommen haben. Für $C$ ist daher jedenfalls die Hauptursache der erhöhten Genauigkeit in der vermehrten Uebung und in der Beherrschung der Eigenthümlichkeiten des Uhrwerks zu suchen.

Im Besonderen entnehmen wir der obigen Tabelle noch, dass die Genauigkeit eines halben Durchgangs (Gruppe I, 15 Contacte) wesentlich geringer als die eines vollen (Gruppe II, 26 Contacte) ist und bedeutend erhöht wird durch die Hinzunahme der anderen Häfte. Zufall ist es wohl, dass die Beobachtungen der Gruppe I genauer sind als die der Gruppe II, obwohl die durch das Wechseln der Beobachter entstehende Störung den umgekehrten Effect befürchten liesse. Allerdings giebt auch die zweite Art der Berechnung als mittlere Fehler für beide Sterngruppen \pm 0.019 und \pm 0.021 ; indessen kann dies davon herrühren, dass zur Reduction der Beobachtungen der Gruppe II auf $1 / 2(C+P)$ der Durchschnittswerth der ganzen Beobachtungsreihe

$$
\pm 1 / 2(C-P)=\mp \text { os.o1 } 1 \text {, }
$$

und nicht die speciellen Abendwerthe angewandt sind.

Die zweite Art der Berechnung zeigt, dass die Helligkeit auf die mittleren Fehler kaum von Einfluss gewesen ist.

\begin{tabular}{c|c|c}
\hline Helligkeit & Mittl. Fehler & Zahl d. Sterne \\
\hline$\geq 6^{\mathrm{m}} \cdot 0$ & $\pm 0^{\mathrm{s}} \mathrm{2} 2 \mathrm{I}$ & $4 \mathrm{I}$ \\
$6^{\mathrm{m}} \mathrm{I}-7^{\mathrm{m}} \cdot 5$ & \pm 0.0201 & I 3 \\
$7.6-8.0$ & \pm 0.0184 & 24 \\
$8.1-8.2$ & \pm 0.0219 & I I \\
$\leq 8^{\mathrm{m}} \cdot 3$ & \pm 0.0228 & 8
\end{tabular}

Nach diesen besonderen Folgerungen wenden wir uns zu der thatsächlich erreichten individuellen Genauigkeit.

Aus dem ganzen Material der Gruppe II folgt:

$$
\varepsilon_{C}= \pm 0.019, \quad \varepsilon_{P}= \pm 0.023 \text {, }
$$

im Durchschnitt: \pm 0.021 ;

aus den Sternen der Gruppe I:

$$
\varepsilon_{1 / 2}(C+P)= \pm 0.019 \text {. }
$$

Das Mittel von \pm 0.020 giebt auf den Aequator reducirt einen mittleren Fehler einer Beobachtung von

$$
\varepsilon= \pm 0.019= \pm 0.28 \text {, }
$$

welcher der Gesammtheit unserer Beobachtungen entspricht.
Schon das ist für Meridianbeobachtungen eine bemerkenswerthe Genauigkeit. Beachtet man indessen, dass die ersten Abende mehr zur Uebung dienten, viel durch Wolken gestört wurden und einen viel weniger befriedigenden Eindruck hinterliessen, als die folgenden Abende, die thatsächlich geringere mittlere Fehler aufweisen, so wird man für diese späteren Abende die Werthe:

$$
\begin{aligned}
& \varepsilon_{C}= \pm 0.016= \pm 0.23 \mathrm{sec} \delta \\
& \varepsilon_{P}= \pm 0.020= \pm 0.28 \mathrm{sec} \delta
\end{aligned}
$$

eher noch als zu hoch gegriffen bezeichnen müssen. Thatsächlich geben die beiden letzten Abende als mittleren Fehler einer Position (aus 63 resp. 62 Sternen):

$$
\varepsilon= \pm 0.0155= \pm 0.22 \sec \delta \text {. }
$$

Bedenkt man nun noch, dass diese Beobachtungen infolge des beständigen Wechselns der Beobachter und der deshalb erforderlichen Verstellung des Oculars, sowie der durch die Einfuhrung der Kappe bedingten Störung noch nicht den höchsten Grad der Genauigkeit besitzen können, so ist man wohl berechtigt anzunehmen, dass ein mittlerer Fehler von \pm 0.25 für einen nur einigermaassen geübten Beobachter ein leicht erreichbarer durchschnittlicher Genauigkeitsgrad ist und dass er aller Voraussicht nach noch tiefer, vielleicht gar bis \pm 0.20 , wird herabgedrückt werden können. In der That hat sich z. B. bei meinen seitherigen Beobachtungen durch Vergleichung zweier Abende (Sept. 20 und 22 ) mit je 56 gemeinsamen Sternen bis $2 u{ }^{\mathrm{m}} \cdot \mathrm{t}$ hinab als mittlerer Fehler einer Beobachtung im Bogen grössten Kreises \pm 0 " I 8 ergeben. Aber auch eine Genauigkeit, wie sie dem oben für $C$ ermittelten Werthe von \pm 0.23 entsprechen würde, ist bisher niemals auch nur annähernd bei Meridianbeobachtungen erreicht, vielmehr ein doppelt so grosser mittlerer Fehler noch als recht erfreulich angesehen worden. Zu den allerbesten Meridianbeobachtungen gehören ohne Frage die mit dem grossen Berliner Meridiankreise angestellten, mit dessen Leistungen sich der uber 60 Jahre alte, vierzöllige Repsold'sche Meridiankreis der hiesigen Sternwarte gar nicht vergleichen lässt. Für den ersteren finden nun Klistner (Untersuchungen über die Eigenbewegungen von 335 Sternen, Veröff. der Bonner Sternwarte Nr. 2, S. 5 A. N. 3392/93) und Battermann (1. c., S. I 2) als mittleren Fehler von $\Delta \alpha \cos \delta \pm 0.33$. Ferner erhält z. B. Küstner für den Bonner neuen Meridiankreis als mittleren Fehler von $\Delta \alpha \cos \delta$ (bei Ausschluss der schwächsten Sterne $9 \cdot \mathbf{m}$ bis 10 m) :

$$
\begin{aligned}
& \text { I 894.3-96.3 土0."5 I } \\
& \text { 1896.3-97.3 } \pm 0.44 \\
& \text { 1897-3-98.3 土0.40. }
\end{aligned}
$$

Danach wird man unseren späteren Beobachtungen fast das doppelte Gewicht der allerersten und mindestens das dreifache sonst als recht gut anerkannter Registrirbeobachtungen zuschreiben dürfen.

Es ist hier nicht der Platz, eine ausfuhrliche Uebersicht der Beobachtungen im Einzelnen zu geben; um aber doch diese etwas wenig anschaulichen mittleren Fehler durch einige concrete Zahlen zu erläutern, möchte ich hier die von jedem Beobachter erhaltenen Endpositionen gegenüberstellen, 
da man aus ihren Unterschieden ein klares Bild der erreichten Genauigkeit erhält. Da die zur Reduction angewandte, als lineare Function der Zeit angesetzte Uhrcorrection sich auf das System $1 / 2(C+P)$ bezieht, wurde den Beobachtungen des einzelnen Beobachters die halbe Differenz: $\pm 1 / 2(C-P)=\mp 0.0 \times 1$ hinzugefügt, wie sie im Mittel der ganzen Reihe erhalten war. Die erste Columne der folgenden Tabelle giebt die Bezeichnung des Sterns nach BD., resp. bei den der Fixirung des Systems dienenden Jahrbuchsternen ihren Namen, die zweite die Helligkeit nach der Potsdamer
Durchmusterung resp. eigenen Schatzungen. Es folgt die Declination für $190 \mathrm{I}$ und darauf in zwei Columnen die Beobachtungsergebnisse, nach den Beobachtern getrennt, mit der Zahl der Einzelwerthe, auf der jede Position beruht. Da die durchschnittliche Zahl far jeden Stern etwa 10 ist, so erkennt man sofort, welche Sterne der Gruppe I, welche der Gruppe II angebören, indem die bei den ersteren gegebenen Zahlen sich nur auf den halben Durchgang beziehen. ${ }^{1}$ ) Endlich folgen die individuellen Unterschiede $C-P$ nach Berücksichtigung des Durchschnittswerthes von - -\$o2 2.

Rectascensionen für r $90 \mathrm{r}$.

\begin{tabular}{|c|c|c|c|c|c|c|c|c|c|c|}
\hline Name & Grösse & & $\delta$ & & & $C$ & Zahl & $P$ & Zahl & Differenz \\
\hline$\gamma$ Herculis & $4 \cdot 0$ & $19^{\circ}$ & $23^{\prime}$ & $16^{h}$ & $17^{\mathrm{n}}$ & ${ }^{m} 33^{5} \times 27$ & 9 & $33^{5} .143$ & 9 & -0.016 \\
\hline$\infty$ Herculis & 4.8 & 14 & 16 & 16 & 20 & 50.755 & 9 & 50.771 & 9 & -0.016 \\
\hline$+15: 3007$ & 7.6 & 15 & 34 & I 6 & 23 & 34.529 & 9 & 34.528 & 9 & +0.001 \\
\hline$\beta$ Herculis & 3.0 & 2 I & 42 & 16 & 25 & 57.810 & .9 & $57 \cdot 767$ & 9 & +0.043 \\
\hline+11.3008 & 4.9 & I I & 42 & 16 & 27 & 58.234 & 9 & $5^{8.2} 5^{6}$ & 9 & -0.022 \\
\hline+17.3051 & 8.5 & 17 & 18 & I 6 & $3^{\circ}$ & 10.1 $3^{6}$ & 4 & 10.140 & 4 & -0.004 \\
\hline+17.3053 & 6.5 & 17 & I 5 & I 6 & 30 & 59.972 & 5 & 59.976 & 5 & -0.004 \\
\hline+16.2983 & 7.9 & 15 & 54 & I 6 & $3^{2}$ & 46.973 & 9 & 46.985 & 9 & -0.012 \\
\hline+17.3067 & 8. I & 17 & & I 6 & 35 & 26.933 & 9 & 26.930 & 9 & +0.003 \\
\hline+15.3040 & 7.4 & I 5 & $5 \circ$ & I 6 & $3^{8}$ & 27.774 & 5 & $27.76_{3}$ & 3 & +0.011 \\
\hline+16.3013 & $5 \cdot 7$ & $\times 5$ & 55 & 16 & 40 & 53.472 & 5 & 53.461 & 3 & +0.011 \\
\hline+18.3237 & 8.3 & 18 & 7 & I 6 & 42 & $30.25^{\circ}$ & 5 & 30.280 & 3 & -0.030 \\
\hline+18.3244 & $7 \cdot 7$ & 18 & 30 & 16 & 44 & $20.20 \mathrm{I}$ & 5 & 20.207 & 4 & -0.006 \\
\hline 49 Herculis & 6.8 & I 5 & 8 & I 6 & 47 & $34.35^{I}$ & 4 & 34.357 & 5 & -0.006 \\
\hline+10.3092 & 4.6 & Io & I9 & I 6 & 49 & 19.365 & 5 & 19.377 & 4 & -0.012 \\
\hline+18.3266 & 5.4 & 1 8 & 35 & 16 & $5 \mathrm{I}$ & 1.062 & 5 & 1.069 & 5 & -0.007 \\
\hline$x$ Ophiuchi & 3.4 & 9 & 32 & 16 & $5^{2}$ & $5^{8.874}$ & 5 & 58.885 & 5 & -0.011 \\
\hline$+17.3^{1} 3^{6}$ & $7 \cdot 7$ & 17 & 50 & 16 & 54 & 41.291 & 4 & 41.280 & 5 & +0.011 \\
\hline$\varepsilon$ Herculis & 4.2 & 31 & 4 & 16 & $5^{6}$ & 30.084 & 5 & $3^{0.076}$ & 5 & +0.008 \\
\hline+14.3178 & 8. I & 14 & 48 & 16 & $5^{8}$ & 23.605 & 5 & 23.607 & 4 & -0.002 \\
\hline 6o Herculis & 5.0 & I 2 & $5^{2}$ & I 7 & $\circ$ & 47.187 & 5 & 47.208 & 5 & $-0.02 \mathrm{I}$ \\
\hline+15.3116 & 8. 1 & I 5 & 20 & I 7 & 3 & 12.452 & 5 & 12.457 & 4 & -0.005 \\
\hline+18.3314 & 8.1 & 18 & 3 & I 7 & 5 & 25.955 & 4 & 25.956 & 4 & -0.001 \\
\hline+10.3165 & $5 \cdot 3$ & 10 & 42 & 17 & 7 & 47.729 & 5 & $47 \cdot 74^{8}$ & 5 & -0.019 \\
\hline$\alpha$ Herculis & var. & 14 & 30 & 17 & 10 & 7.972 & 5 & 7.933 & 5 & +0.039 \\
\hline+16.3139 & 8. I & 16 & 46 & 17 & I I & 55.065 & 4 & 55.068 & 5 & -0.003 \\
\hline$+11.3^{156}$ & 5.0 & Io & $5^{8}$ & 17 & I 3 & 57.662 & 5 & 57.668 & 5 & -0.006 \\
\hline$+16.315 \mathrm{I}$ & 7.5 & I 5 & 58 & $\mathbf{I} 7$ & r 5 & $33 \cdot 526$ & 5 & 33.524 & 4 & +0.002 \\
\hline+16.3163 & 6.5 & r 6 & 50 & 17 & 17 & 8.439 & 5 & 8.438 & 5 & +0.001 \\
\hline+15.3182 & 7.8 & I 5 & 38 & 17 & 20 & 57.277 & II & 57.283 & I I & -0.006 \\
\hline+16.3188 & 7.5 & 16 & 32 & 17 & 23 & 7.223 & 12 & 7.221 & 10 & +0.002 \\
\hline+16.3197 & 8.2 & I 6 & 2 & 17 & 25 & 24.689 & I 2 & 24.703 & I I & -0.014 \\
\hline+16.3209 & 7.2 & I 6 & 54 & 17 & 27 & 29.776 & 12 & 29.767 & 12 & +0.009 \\
\hline$\alpha$ Ophiuchi & 2.5 & 12 & 38 & 17 & 30 & 20.287 & I 3 & 20.293 & I 3 & -0.006 \\
\hline+14.3289 & 8.0 & I 4 & 55 & $\mathbf{I} 7$ & 32 & 32.955 & 13 & $32.95^{\circ}$ & I 3 & $+0: 005$ \\
\hline+18.3428 & 7.9 & 18 & 3 & 17 & 35 & I. I 31 & I 3 & I.I I I & I 3 & +0.020 \\
\hline$+16.3^{2} 5^{6}$ & 5.7 & 16 & 0 & 17 & 37 & 32.017 & 12 & 32.014 & I 2 & +0.003 \\
\hline+19.3416 & 8.6 & 19 & $\mathbf{I}$ & 17 & 40 & I 3.259 & 12 & 13.265 & I 2 & -0.006 \\
\hline+17.3334 & 5.8 & 17 & 44 & 17 & 42 & 45.797 & I 3 & 45.807 & 12 & -0.010 \\
\hline+12.3300 & 8.3 & 12 & 48 & 17 & 45 & I 5.440 & 10 & 15.437 & 10 & +0.003 \\
\hline+15.32 .92 & 6.6 & 15 & $2 \mathrm{I}$ & 17 & 47 & 30.444 & I 2 & 30.440 & I 2 & +0.004 \\
\hline
\end{tabular}

1) Bei den Jahrbuchsternen sind die gefundenen Correctionen an die mit den vvorläufigen Verbesserungen * versehenen Positionen für 1901.0 angebracht, so dass sie die Eigenbewegung bis 1901.0 schon in sich enthalten; alle übrigen gelten für die Epoche 1901.53. 


\begin{tabular}{|c|c|c|c|c|c|c|c|c|c|c|}
\hline Name & Grösse & $\delta$ & $\xi$ & & & $C$ & Zahl & $P$ & Zahl & Differenz \\
\hline$+15: 3297$ & 8. 1 & $15^{\circ}$ & $49^{\prime}$ & $17^{\mathrm{h}}$ & $49^{\mathrm{m}}$ & $17^{s} \cdot 43^{1}$ & 13 & $17^{5} \cdot 43^{1}$ & 13 & 0.000 \\
\hline+20.3616 & 8.3 & 20 & 4 & 17 & 51 & 14.597 & 12 & 14.577 & 12 & +0.020 \\
\hline+15.3309 & 7.9 & I 5 & 25 & I 7 & 53 & 34.286 & 12 & 34.284 & 12 & +0.002 \\
\hline+16.3335 & $4 \cdot 7$ & 16 & 45 & 17 & 55 & 38.971 & 13 & $3^{8.966}$ & 13 & +0.005 \\
\hline+16.3347 & $7 \cdot 7$ & 16 & 48 & 17 & 58 & 18.705 & I3 & 18.708 & 13 & -0.003 \\
\hline+15.3354 & 7.6 & 15 & 38 & 18 & o & 51.377 & 12 & $51 \cdot 360$ & 12 & +0.017 \\
\hline o Herculis & 4.1 & 28 & 44 & 18 & 3 & 40.817 & I 3 & 40.8 I 5 & $\mathbf{1} 3$ & +0.002 \\
\hline+16.3393 & 7.9 & & & I 8 & 6 & I 6.963 & 12 & $16.93^{8}$ & 12 & +0.025 \\
\hline+16.3405 & 6.9 & 16 & 15 & I 8 & 8 & 50.034 & I 2 & $50.03^{6}$ & 12 & -0.002 \\
\hline+15.3415 & 8.2 & 15 & 19 & I 8 & $\mathbf{x}$ & $25 \cdot 3^{21}$ & I I & $25.34^{\circ}$ & I I & -0.019 \\
\hline$+18.3^{623}$ & 6.5 & 18 & & r 8 & I 3 & 46.577 & I I & 46.554 & I I & +0.023 \\
\hline+17.3536 & 7.9 & 17 & 20 & I 8 & 16 & $21.23^{2}$ & I I & $21: 231$ & I I & +0.001 \\
\hline 109 Herculis & 4.1 & 21 & 42 & 18 & 19 & 28.717 & I I & 28.697 & 10 & +0.020 \\
\hline+19.3643 & 8.1 & 19 & 7 & 18 & 22 & 3.043 & I I & $3.03^{6}$ & 9 & +0.007 \\
\hline+14.3546 & 7.6 & 14 & $5^{6}$ & I 8 & 24 & 23.482 & II & $23.49 \mathrm{I}$ & I I & -0.009 \\
\hline+16.3529 & $5 \cdot 7$ & 16 & $5^{2}$ & 18 & 26 & 40.200 & 8 & 40.203 & 8 & -0.003 \\
\hline+18.3728 & 7.0 & 18 & 51 & 18 & 28 & $55 \times 56$ & 9 & 55.154 & 9 & +0.002 \\
\hline$+18.3740^{\circ}$ & 6.1 & 18 & 8 & 183 & 30 & 51.120 & II & 51.109 & I I & $+0.01 \mathrm{I}$ \\
\hline+17.3679 & $7 \cdot 5$ & 17 & 31 & 183 & 35 & 31.292 & 4 & $3^{1.298}$ & 5 & -0.006 \\
\hline+17.3687 & 8.0 & 17 & 35 & I 8 & 37 & 13.918 & 5 & 13.937 & 6 & -0.019 \\
\hline+19.3762 & $7 \cdot 5$ & 19 & 22 & 18 & 38 & 55.981 & 5 & 55.973 & 6 & +0.008 \\
\hline I 10 Herculis & 4.5 & 20 & 27 & 18 & 41 & 24.047 & 5 & 24.036 & 8 & $+0.01 \mathrm{I}$ \\
\hline$+18.3^{823}$ & 4.4 & 18 & 4 & 18 & 42 & 38.914 & 3 & $3^{8.908}$ & 6 & +0.006 \\
\hline+19.3798 & 6.3 & 19 & 13 & 18 & 44 & 34.237 & 7 & 34.239 & 5 & -0.002 \\
\hline$\beta$ Lyrae & var. & 33 & I 5 & 18 & 46 & $25 \cdot 502$ & 7 & 25.489 & 5 & +0.013 \\
\hline+17.3761 & 8.4 & 17 & 14 & 18 & 48 & 34.700 & 7 & 34.676 & 5 & +0.024 \\
\hline+17.3779 & 5.9 & 17 & 59 & 18 & 51 & 44.021 & 7 & $44.04^{I}$ & 5 & -0.020 \\
\hline+17.3799 & 5.6 & 17 & 14 & I 8 & 53 & 50.378 & 4 & 50.406 & 3 & -0.028 \\
\hline$\varepsilon$ Aquilae & $4 \cdot 3$ & I 4 & $5^{6}$ & 18 & 55 & 7.724 & 7 & $7.73^{1}$ & 5 & -0.007 \\
\hline+18.3922 & 7.1 & I 8 & 59 & I 8 & 57 & $36.93 \circ$ & 5 & $3^{6.94}$ I & 7 & -0.011 \\
\hline$+17 \cdot 3^{8} 3^{8}$ & 7.1 & I 7 & 8 & 18 & 59 & 9.622 & 5 & 9.643 & 7 & $-0.02 \mathrm{I}$ \\
\hline$\zeta$ Aquilae & $3 \cdot 3$ & 13 & 42 & 19 & $\circ$ & $51.55^{\circ}$ & 5 & 51.564 & 7 & -0.014 \\
\hline+16.3741 & 8.3 & 16 & 55 & 19 & 2 & 43.014 & 4 & 43.023 & 7 & -0.009 \\
\hline+18.3967 & 8.2 & I 8 & 27 & 19 & 4 & 32.447 & 5 & $3^{2} \cdot 443$ & 7 & +0.004 \\
\hline+15.3721 & $7 \cdot 7$ & I 5 & $3^{8}$ & 19 & 6 & 42.4 I 5 & 7 & 42.4 I 5 & 5 & 0.000 \\
\hline+17.3890 & 8.0 & 17 & 20 & 19 & 8 & 34.184 & 7 & 34.173 & 5 & +0.011 \\
\hline+16.3792 & 8.0 & 16 & 15 & 19 & 10 & $25 \cdot 378$ & 7 & 25.385 & 5 & -0.007 \\
\hline$\infty$ Aquilae & 5.4 & 11 & 25 & I9 & I 3 & 10.125 & 7 & 10.125 & 5 & 0.000 \\
\hline+16.3819 & 8.3 & 16 & 40 & I 9 & 16 & 0.622 & 3 & 0.599 & 7 & +0.023 \\
\hline+17.3943 & $7 \cdot 3$ & 17 & 34 & I 9 & 17 & 39.128 & 4 & 39.104 & 6 & +0.024 \\
\hline$+11.3^{8} 33$ & $5 \cdot 3$ & II & 44 & 19 & 20 & $14.95^{\circ}$ & 5 & $14.97^{2}$ & 7 & -0.022 \\
\hline+19.4015 & 5.9 & 19 & 54 & 19 & $2 \mathrm{I}$ & 53.838 & 5 & 53.806 & 7 & +0.032 \\
\hline+19.4028 & 7.2 & 20 & 3 & 19 & 23 & 34.901 & 4 & 34.879 & 7 & +0.022 \\
\hline$\beta$ Cygni & 3.2 & 27 & 45 & 19 & 26 & 43.729 & 7 & 43.712 & 4 & +0.017 \\
\hline+10.3953 & 8.4 & 10 & 34 & I9 & 28 & 39.284 & 6 & 39.27 I & 2 & +0.013 \\
\hline+19.4063 & 5.2 & 19 & 34 & 19 & 30 & 13.982 & 6 & 13.980 & 4 & +0.002 \\
\hline$+15.3^{877}$ & 7.4 & I 5 & 40 & 19 & 31 & $5^{2.655}$ & 5 & $5^{2.6} 39$ & 2 & +0.016 \\
\hline+19.4092 & 8.3 & 19 & 39 & 19 & 34 & 3.968 & 7 & $3.93^{8}$ & 4 & +0.030 \\
\hline+17.4048 & 4.6 & 17 & 15 & 19 & $3^{6}$ & 36.126 & 4 & 36.127 & 6 & -0.001 \\
\hline+17.4059 & 8.0 & 17 & 44 & 19 & 38 & 22.526 & 4 & 22.5 I 8 & 7 & +0.008 \\
\hline+18.4216 & 7.0 & 18 & $2 \mathrm{I}$ & 19 & 40 & 6.935 & 4 & 6.935 & 3 & 0.000 \\
\hline$\delta$ Sagittae & 3.9 & I 8 & 17 & 19 & 42 & 58.371 & 5 & $5^{8.362}$ & 7 & +0.009 \\
\hline$\alpha$ Aquilae & 1.2 & 8 & $3^{6}$ & 19 & 45 & 57.173 & 3 & 57.168 & 5 & +0.005 \\
\hline+15.3970 & 7.8 & 16 & 0 & 19 & 48 & 24.209 & 7 & 24.218 & 5 & -0.009 \\
\hline$\beta$ Aquilae & 3.9 & 6 & 9 & 19 & $5^{\circ}$ & 26.978 & 7 & 27.002 & 5 & -0.024 \\
\hline+18.4315 & 8.0 & I 8 & 55 & 19 & $5^{2}$ & $5 \cdot 547$ & 7 & $5 \cdot 528$ & 5 & +0.019 \\
\hline
\end{tabular}




\begin{tabular}{|c|c|c|c|c|c|c|c|}
\hline Name & Grösse & $\delta$ & $C$ & Zahl & $P$ & Zahl & Differenz \\
\hline$\gamma$ Sagittae & 3.8 & $19^{\circ}$ I $3^{\prime}$ & $19^{\mathrm{h}} 54^{\mathrm{m}} 21^{\mathrm{s}} 234$ & 7 & $\begin{array}{l}211_{2} 3^{8} \\
20,278\end{array}$ & 5 & $\begin{array}{l}-0.004 \\
-0.006\end{array}$ \\
\hline
\end{tabular}

Die Anordnung der Werthe nach der Helligkeit und nach der Rectascension war schon oben besprochen. Wir berechnen jetzt noch den mittleren Fehler einer Differenz $C-P$, unabhängig von der Zahl der Beobachtungen, auf der sie beruht, und finden: \pm 0.50144 . Hieraus schliessen wir, dass der mittlere Fehler einer definitiven Rectascension $1 / 2(C+P)$ beträgt:

$$
\pm 0.0072= \pm 0.10 \sec \delta \text {. }
$$

Da wir oben als mittleren Fehler einer Beobachtung 土0.28 sec $\delta$ gefunden hatten, ist eine Reduction beinahe $\sqrt{10}$ entsprechend, wie es die Zahl der Beobachtungen erfordern würde, eingetreten; auch das wirft ein günstiges Licht auf die Leistungsfähigkeit der Methode. Dass der mittlere Fehler einer Differenz $C-P$ fur die Sterne der Gruppe I kleiner ausfallt ( \pm 0.0134$)$ als für die der Gruppe II $( \pm 0.0152)$, liegt in der Natur der Sache.

Fassen wir zum Schluss die Ergebnisse kurz zusammen, so können wir wohl sagen, dass sie den Erwartungen voll und ganz entsprochen haben. Die schon bei der Einführung des Repsold'schen Mikrometers erwartete Beseitigung des schädlichen Einflusses der persönlichen Gleichung und ihrer Schwankungen hat sich jetzt bestätigt. Daneben ist aber durch die Einfuihrung des Uhrwerks die Beobachtungsart eine wesentlich bequemere und dementsprechend die $\mathrm{Ge}$ nauigkeit eine bei Meridianbeobachtungen bisher nicht annähernd erreichte geworden. Allerdings beziehen sich unsere Beobachtungen nur auf einen schmalen Gürtel, und über den Einfluss verschiedener Declinationen muss ein endgültiges Urtheil noch abgewartet werden. Schon jetzt aber wird man bei allen Aufgaben differentieller Art, bei denen eine besondere Constanz der persönlichen Gleichung und eine Elimination der Helligkeitsgleichung erforderlich ist, der Ein. führung des Repsold'schen Mikrometers mit Uhrwerk mit grossen Erwartungen entgegensehen dürfen. Von dieser Art sind aber heutzutage fast alle Beobachtungen am Meridiankreise. Denn auf der möglichst differentiellen Anordnung gegenüber dem früher oft üblichen planlosen Beobachten irgend welcher Sterne beruht ja in der Hauptsache die wesentlich gesteigerte Genauigkeit der Meridianbeobachtungen in den letzten Jahrzehnten. Selbst die völlige Trennung der beiden Coordinaten, wie sie bei Anwendung des Uhrwerks vielleicht rathsam sein möchte, ist daher für viele Fragen kaum als Nachtheil anzusehen; denn die Möglichkeit, für jede einzelne Coordinate in kurzer Zeit ein umfangreicheres und gleichförmigeres Beobachtungsmaterial zu sammeln, giebt der Discussion der Fehlerquellen eine viel grössere Sicherheit, wie ja auch in unserem Falle darauf im Wesentlichen die liberzeugende Kraft unserer Folgerungen beruht.

Zunächst habe ich eine erneute Beobachtung der vor einem Jahre nach der Registrirmethode beobachteten Rectascensionen der Eros-Sterne, sodann der Liste der 2798 Gill'schen Zodiacalsterne begonnen, um an einem grösseren Material die Leistungsfähigkeit der Methode zu erproben. Dann aber soll im nächsten Frühjahr die noch wesentlich wichtigere Anwendung auf Sonnenbeobachtungen und in Verbindung damit auf die Bestimmung des Aequinoctiums folgen; die bisherigen Versuche an Sonne und Mond lassen auch hier einen Erfolg der neuen Methode mit Sicherheit erhoffen.

Dass sich die Methode durch das Wegfallen der persönlichen Fehlerquellen und die erhöhte Genauigkeit auch zur Bestimmung von Fixsternparallaxen im Sinne der Kapteyn' schen Arbeit (Leidener Annalen, Bd. VII) eignen dürfte, ist wohl einleuchtend, sowie dass sie auch für Längenbestimmungen, bei denen schon das Repsold'sche Mikrometer in seiner gewöhnlichen Anwendung sich sehr bewährt hat, eine weitere Steigerung der Genauigkeit erwarten lăsst.

So darf man wohl sagen, dass durch ausgedehnte Anwendung des Repsold'schen Mikrometers in Verbindung mit dem Uhrwerk den Beobachtungen am Meridiankreise manche Gebiete werden zurückerobert werden, welche ihm in den letzten Jahrzehnten durch Heliometer und Photographie schon entrissen schienen.

Königsberg, I90 I Nov. 12.

Fritz Cohn.

\section{Observations de petites planètes et de comètes}

faites à l'Observatoire d'Alger à l'équatorial coudé de ${ }^{\mathrm{m}} \mathbf{3}^{1} 8$ par MM. Rambaud et $S y$.

\begin{tabular}{|c|c|c|c|c|c|c|c|c|c|c|c|}
\hline Date & T. m. Alger & $\Delta \alpha$ & $\Delta \delta$ & Cp. & Obs. & $\alpha$ app. & $\log p . A$ & $\delta$ app. & $|\log p .4|$ & Red. ad l. app. & * \\
\hline \multicolumn{12}{|l|}{1900} \\
\hline Mai & $10^{h} 30^{m} 45^{s}$ & $+1^{m} 10^{s} 67$ & $+0^{\prime} 24: 5$ & 15.10 & $\mathbf{R}$ & $16^{\mathrm{h}} 7^{\mathrm{m}} 5^{8 \mathrm{~s}} .8 \mathrm{I}$ & $9.504 n$ & $-13^{\circ} 28^{\prime} 31^{\prime \prime} 7$ & 0.805 & $+3.50-8.13$ & $\mathbf{I}$ \\
\hline 5 & I I I I & +19.67 & +028.0 & 15.10 & $\mathbf{S}$ & $\mathrm{I} 6757.8 \mathrm{I}$ & $7 n$ & -132828.2 & 0.814 & $.50-8.3$ & I \\
\hline 9 & ro 1646 & -0 $6.4^{2}$ & $+8 \quad 1.9$ & 15.12 & $\mathbf{R}$ & $\begin{array}{lll}\text { I } & 4 & 39.47\end{array}$ & $9.49^{2} \mathrm{n}$ & $-1317 \quad 12.2$ & 0.805 & $+3.57-8.3$ & 2 \\
\hline 9 & 104022 & -0 7.36 & +84.1 & 15.12 & $\mathbf{S}$ & $1643^{8.53}$ & $9.43^{2} \mathrm{n}$ & $-1317 \quad 10.0$ & 0.812 & $+3.57-8.3$ & 2 \\
\hline 10 & $\begin{array}{lll}10 & 29 & 37\end{array}$ & $-0 \quad 59.46$ & +1050.3 & $\times 5.10$ & $\mathbf{S}$ & 16346.44 & $9.44^{8} n$ & -131423.8 & 0.810 & $+3.5^{8}-8.3$ & 2 \\
\hline 10 & $\begin{array}{lll}10 & 42 & 49\end{array}$ & $-0 \quad 59.86$ & + Io 5 I.I & 15.10 & $\mathbf{R}$ & 16346.04 & $9.410_{n}$ & -13 I4 23.0 & $0.8 \mathrm{I}_{4}$ & $+3.5^{8}-8.3$ & 2 \\
\hline
\end{tabular}

